\title{
Psychometric properties of the Birth Satisfaction Scale-Revised (BSS-R) for US mothers
}

Celestina Barbosa-Leiker ${ }^{\mathrm{a}, \mathrm{b}^{*}}$, Susan Fleming ${ }^{\mathrm{a}}$, Caroline J. Hollins Martin ${ }^{\mathrm{c}}$, and Colin R. Martin ${ }^{\mathrm{d}}$

${ }^{a}$ College of Nursing, Washington State University, Spokane, WA, USA; ${ }^{b}$ Department of Psychology, Washington State University, Pullman, WA, USA; 'School of Nursing, Midwifery and Social Care, University of Salford, Greater Manchester, England, UK; ${ }^{\mathrm{d}}$ Faculty of Society and Health, Buckinghamshire New University, Uxbridge, England, UK

*Corresponding author: Celestina Barbosa-Leiker, Washington State University, College of Nursing, PO Box 1495 Spokane, WA, USA, 99210-1495; Phone: +1-509-324-7477; Fax: +1509-324-7341; Email: celestina@wsu.edu.

Word count: 2994

Running head: Birth Satisfaction of US Mothers 


\begin{abstract}
Objective: The aims of this study were to continue the scale development process of the Birth Satisfaction Scale-Revised (BSS-R) by refining the scale to make it culturally relevant for US participants, examining the factor structure of the BSS-R, and describing the level of birth satisfaction in a sample of US mothers. Background: The Birth Satisfaction Scale (BSS) was developed in the UK to assess satisfaction of the childbearing women's experiences of labor and its outcomes. One of the goals of the development of the BSS was to make comparisons across cultures. Methods: One-hundred and eighty-one first time US mothers participated in this study. Confirmatory factor analysis was used to examine a 1-factor higher-order model containing 3 lower-order factors. The higher-order factor was hypothesized to be Experience of childbearing; the lower-order factors were hypothesized as Stress, Quality of Care, and Women’s attributes.
\end{abstract} Results: The results of the higher-order factor model indicated good fit, $\chi^{2}=37.72, p=.22$; comparative fit index $(\mathrm{CFI})=.99$; root mean square error of approximation $(\mathrm{RMSEA})=.03$; standardized root mean square residual $(\mathrm{SRMR})=.04$. Cronbach's $\alpha$ indicated the subscales and total scale were reliable for the US sample ( $\alpha$ ranged from .74 to .89). The BSS-R total score was 15.52 (SD=8.35), and the stress, quality of care, and women's attributes subscales were 7.15 ( $\mathrm{SD}=3.80), 4.61(\mathrm{SD}=3.83)$ and $3.79(\mathrm{SD}=2.26)$, respectively. Conclusion: The BSS-R can provide maternal health professionals and researchers with an instrument to quantify childbearing women's birthing satisfaction, which in turn can assist in heightening the overall patient care experience.

Keywords Birth satisfaction, Intrapartum care, Maternal-Infant care, Psychometrics 


\section{Introduction}

Recently, the Institute of Healthcare Improvement (IHI) established a framework to heighten the patient care experience, improve overall health and reduce costs. This is known as “The Triple Aim” (IHI, 2014). Today, there is a national collaborative effort by U.S. healthcare providers and policy makers to meet the goals of the “Triple Aim” by improving patients' health and healthcare experiences. In the 1950s the U.S. claimed an unrivaled success in regards to their high ranking of health outcomes, including maternal-newborn, among other industrialized nations, primarily in Europe. However, over the past several decades, a spiraling decline of overall health outcomes including maternal-newborn outcomes has been observed (Bezruchka, 2012; Clark, Belfort, Byrum, Meyers, \& Perlin, 2008). Maternity care in the US is dominated by costly procedures and ritual-based intensive clinical practice; yet, maternal-newborn outcomes remain poor when compared to other industrialized nations (Bezruchka, 2012; MacDorman \& Matthews, 2009; Stones \& Arulkumaran, 2014). Numerous maternal care experts consider this US phenomenon an American paradox of “doing more while accomplishing less” (Childbirth Connections, 2014). The cesarean rate continues to remain high at $32.8 \%$ and contributes to the deteriorating national ranking (Bezruchka, 2012; MacDorman \& Matthews 2009; Martin, Hamilton, Osterman, Curtin, \& Matthews, 2013). In 2012 over 98\% of births occurred in the hospital, however, only 7.6\% were attended by certified nurse midwifes (Martin et al., 2013). Midwifery-led care is known for their attention to low-procedure care and acclaims positive birthing outcomes (Renfrew et al., 2014). US maternal care follows a business fee-based model, however for low income women, $42 \%$ of maternal care is paid for by the government run Medicaid program. The US Affordable Care Act encourages high quality affordable care, which includes increasing the use of midwifery services by paying them $100 \%$ of the physician rate, replacing a 65\% rate used in prior years (Sakala, 2010). 
Notwithstanding these comparatively poor outcomes, in 2008 maternal pregnancy and delivery care was noted to be the most expensive hospital stay condition in the US, surpassing coronary artery disease (Weir \& Andrews, 2011). Furthermore, hospitals cannot offer high quality maternal birth care unless childbearing women are satisfied with their experience (Declercq, Sakala, Corry, Applebaum, Herrlich, 2013). Birth satisfaction refers to a woman’s perception and satisfaction with her intrapartum care (Hollins Martin \& Martin, 2014). Birth satisfaction integrates diverse content, such as feeling in control, coping well during labor, and being treated with respect (Hollins Martin \& Fleming, 2011; Fleming \& Vandermause, 2011). As high quality healthcare cannot be achieved unless the patient is indeed satisfied with their care (Mahon, 1996), it is essential for the childbearing woman to be satisfied with her intrapartum care in order for high quality maternal birth care to be achieved.

In order to quantitatively measure birth satisfaction, the Birth Satisfaction Scale (BSS) was developed to assess satisfaction (or dissatisfaction) of the childbearing women's experiences of labor and its outcomes (Hollins Martin, Snowden, \& Martin, 2012). By reviewing and transcribing birth satisfaction and dissatisfaction expressions from women's birth experiences found in the literature, three themes were posited to describe birth satisfaction: quality of care provision, women’s personal attributes, and stress experienced during labor (Hollins Martin \& Fleming, 2011). The perceptions of birth satisfaction and dissatisfaction were then made into declarative statements: Likert-type items with response categories corresponding to levels of agreement. The aim of this evidence-based 30-item scale was to capture the multi-faceted concept of birth satisfaction in a general way so that healthcare professionals could measure perceptions of intrapartum quality of care. 
An analysis of narrative text that was written by postnatal women in the West of Scotland who simultaneously took the BSS (Hollins Martin, Snowden, \& Martin, 2012) supported the themes that had emerged from the literature (Hollins Martin \& Fleming, 2011). Hollins Martin, Snowden, and Martin (2012) found exemplars of the themes of quality of care provision, women's personal attributes, and stress experienced during labor: the birth going as planned, being supported, and being in control, respectively. These exemplars found in the mothers' narratives demonstrate generalizability of the BSS themes found in the literature.

Most recently, the factor structure, validity, and reliability of the BSS were examined (Hollins Martin \& Martin, 2014). It was found that a 3-factor model of quality of care provision, women's personal attributes, and stress experienced during labor did not fit the data, nor did a single, general factor of birth satisfaction. Upon inspection of poor factor loadings (crossloading, loading < .30, etc.), the BSS was reduced to a 10-item scale. This revised BSS (BSS-R) resulted in a good-fitting 3-factor model. However, a 1-factor higher-order model of birth satisfaction containing 3 lower-order factors (quality of care provision, women’s personal attributes, and stress experienced during labor) proved excellent fit and mapped directly onto the previous work on the BSS (Hollins Martin, Snowden, \& Martin, 2012; Hollins Martin \& Fleming, 2011). The BSS-R demonstrated divergent validity with mother's age, known-groups validity based on normal vs. non-normal deliveries (e.g., forceps and ventouse childbirth, breech birth) and reliability (internal consistency) for postnatal mothers in the West of Scotland (Hollins Martin \& Martin, 2014). Note that there are no conceptually comparable birth satisfaction scales developed in the US for postpartum mothers.

One of the primary goals of the development of the BSS was to be able to make comparisons across cultures (Hollins Martin, Snowden, \& Martin, 2012). In order to do so, the 
psychometric properties of the BSS-R need to be examined with participants in other countries. In addition, further psychometric support of the BSS-R is needed to ensure that previous findings were not sample-dependent. We were interested in how mothers who had not yet experienced birth prepared for their first birth and how satisfied they were. Therefore, the aims of this study were to continue the scale development process of the BSS-R by 1) refining the scale to make it culturally relevant for US participants, 2) examining the factor structure of the BSS-R in a sample of first-time US mothers, and 3) describing the level of birth satisfaction in a sample of first-time US mothers.

\section{Method}

\section{Participants}

Using a cross-sectional, descriptive design, first time mothers were recruited electronically to participate in a comprehensive survey. Participant inclusion criteria included: women, 18 years or older, English speaking, and had given birth in a USA or UK hospital or birthing center in the past 5 years (only US data was used for this study). This study received certification of exemption from our university's Institutional Review Board.

One-hundred and eighty-one US mothers participated in this study. Participants were primarily Caucasian (95.65\%) with a mean age of $28.15(\mathrm{SD}=4.50)$ (mean age at reported delivery was $25.22, \mathrm{SD}=4.16$ ) with an associate degree or some college (31\%) or a Bachelor's degree (40.9\%). Most were currently married (90.6\%) or living with a partner (7.9\%), married at the time of their first birth (84.7\%) or living with a partner (11.3\%), and reported that this first birth was planned (74.4\%). Vaginal delivery was reported for $75.9 \%$ of the sample, with $24.2 \%$ reporting cesarean (of which 1.4\% reported planned cesarean). The first birth occurred within the past year for $31.9 \%$ of the sample, and within the past 4 years for $68.8 \%$. Birth is considered a 
sentinel event and research has confirmed that mothers can recall their birthing experience even twenty years later with incredible accuracy (Simkin, 1991).

\section{Materials and procedure}

The BSS-R is a 10-item scale that was developed in the UK from the original 30-item BSS (Hollins Martin \& Martin, 2014). The BSS-R assesses women’s perceptions of birth in order to determine women's satisfaction of the childbearing experience (Hollins Martin \& Fleming, 2011; Hollins Martin \& Martin, 2014; Hollins Martin, Snowden, \& Martin, 2012). The BSS-R is a Likert-type scale where participants are asked to rate their level of agreement with each item $(1=$ Strongly Disagree, 2 = Disagree, 3 = Neither Agree or Disagree; $4=$ Agree; $5=$ Strongly Agree). Four of the items are reverse-coded (e.g., "I found giving birth a distressing experience”). Three primary changes were made to the BSS-R to make it culturally relevant to US mothers. First, the term "midwife" was changed to "midwife/nurse" in order to make it applicable to the US healthcare provider in the role of the UK midwife. Next, we simply included both spellings of "labour" in the scale items (e.g., "I was not distressed at all during labour/labor"). Finally, we added an item at the end of the scale that used a different term for "unscathed" ("I came through childbirth virtually unscathed"). We thought that this term was not often used in the US so we included the item "I came through childbirth virtually unharmed." For this final change, we analyzed the means across both items to see if there was statistically significant difference in how the US mothers answered these two survey items. Note that while the entire 30-item scale was given to the participants in order to make this study consistent with the previous BSS and BSS-R psychometric studies, our aim was to examine the 10-item BSS-R.

Following informed consent, volunteer participants anonymously provided information about their demographic characteristics, birthing attributes, birthing satisfaction, and current 
depressive symptoms. Participants were recruited from personal and professional contacts via electronic linkages (e.g., Facebook,Twitter, email). As the participant accessed the electronic link, a website appeared that provided information about the study and their role as a participant. At the bottom of the page the participant was given the option to consent or not to consent. If the participant consented to participate in the study, she was allowed to access the questionnaires electronically. If she chose not to consent, she was then transferred to a page which thanked her for her consideration. The data was captured using Qualtrics, an online survey database, over the course of 3 months

\section{Statistical analyses}

Preliminary item analyses included the inspection of the item distributions and utilization of the response categories. A paired-samples t-test using SPSS version 22 (Armonk, NY: IBM Corp) was used to examine potential mean differences across the items "I came through childbirth virtually unscathed" and "I came through childbirth virtually unharmed."

Confirmatory factor analysis (CFA) with robust maximum likelihood estimation and fullinformation MLE to estimate missing data was used to examine a 1-factor higher-order model containing 3 lower-order factors. Consistent with Hollins Martin and Martin, (2014), the higherorder factor was hypothesized to be Experience of childbearing; the lower-order factors were hypothesized as Stress (4 items), Quality of Care (4 items), and Women's attributes (2 items). Model fit was examined using the following criteria: a nonsignificant $\chi^{2}$, comparative fit index $(\mathrm{CFI}) \geq 0.90$, root mean square error of approximation (RMSEA) $\leq 0.080$ and standardized root mean square residual (SRMR) $\leq 0.080$ (Brown, 2006). $P$-values (two-tailed) of $\leq .001$ were used to indicate statistically significant factor loadings. Mplus version 7.11 (Muthén \& Muthén, 19982013) was used for all factor analyses. Cronbach's $\alpha$ was used to examine the internal 
consistency among the sub-scales (lower-order factors) and the total scale. Known-groups analysis using Independent-samples Mann-Whitney U tests comparing birth satisfaction across delivery type (vaginal vs. cesarean) were performed. Lastly, discriminant analysis using Pearson correlations between mother's age at delivery with birth satisfaction was performed. Reliability, known-groups analysis and discriminant analysis was conducted in SPSS version 22 (Armonk, NY: IBM Corp).

\section{Results and discussion}

The BSS-R items demonstrated normal distributions (skewness $<2$, kurtosis $<5$ ) and all response categories were utilized by the participants. The paired-samples $t$-test indicated a statistically significant difference between the "unscathed" item $(\mathrm{M}=3.00, \mathrm{SD}=1.36)$ compared to the "unharmed" item $(\mathrm{M}=2.57, \mathrm{SD}=1.28), t(180)=5.82, p<.001$. Therefore, we chose to use the item "I came through childbirth virtually unharmed" in the factor analysis.

The results of the 1-factor higher-order model containing 3 lower-order factors indicated good model fit, $\chi^{2}=37.72, p=.22$; CFI $=.99 ;$ RMSEA $=.03$; SRMR $=.04$. Table 1 presents the standardized factor loadings for the BSS-R factor model. All items and lower-order factors loaded significantly at $p<.001$. Cronbach's $\alpha$ indicated that the subscales and total scale were reliable for the US sample (stress $=.75$, quality of care $=.85$, women's attributes $=.74$, total BSS-R $=.89$ ). Recoding the BSS-R to a 0-4 scale (Hollins Martin \& Martin, 2014) allowed us to examine the total scores and standard deviations of the BSS-R and 3 sub-scales. The BSS-R total score was 15.52 ( $\mathrm{SD}=8.35$ ), the stress, quality of care, and women's attributes sub-scales were $7.15(\mathrm{SD}=3.80), 4.61(\mathrm{SD}=3.83)$ and $3.79(\mathrm{SD}=2.26)$, respectively. Known-groups analysis using Independent-samples Mann-Whitney U tests for vaginal (76\% of the sample) vs. cesarean deliveries resulted in lower values on the total BSS-R $(\mathrm{U}=3963.00 p<.001)$ and women's 
attributes sub-scale for mothers who had vaginal deliveries ( $\mathrm{U}=4239.50 p<.001)$, as well as lower stress values (note that higher scores relate to greater satisfaction) $(\mathrm{U}=4198.00 p<.001$ ). There were no differences across delivery type on the quality of care sub-scale $(\mathrm{U}=3244.50 p=$ .276). Discriminant analysis indicated that mother's age at delivery was not related to the total BSS-R $(r=-.15, p=.07)$, stress sub-scale $(r=-.01, p=.95)$, or women's attributes sub-scales $(r$ $=-.15, p=.06)$, but older mothers reported lower quality of care $(r=-.24, p=.002)$.

As expected, there was a difference is responses when participants were asked if they came through childbirth unharmed vs. unscathed. While almost identical results were found when the "unscathed" item was used in place of the "unharmed" item in the factor analysis (data not shown), we recommend US researchers and health professionals use the item "I came through childbirth virtually unharmed" in order to get a potentially more accurate BSS-R score.

This study offers additional psychometric support for the BSS-R as applied to a US sample. The BSS-R 1-factor higher-order model containing 3 lower-order factors fit the US data well, with high consistency in what was found in a UK sample (Hollins Martin \& Martin, 2014). Our findings support the computation of 3 subscale scores along with a total BSS-R score to capture birth satisfaction. The 3 subscales and total BSS-R were also reliable for this US sample. Of note is the large discrepancy between the average total score of the quality of care sub-scale in our US sample (4.61) and a recent UK sample (13.76), as well as differences in satisfaction across deliver type (i.e., known-groups analysis) (Hollins Martin \& Martin, 2014). These disparate findings may reflect health service provision and delivery choice options between the US and the UK. Maternity care in the US reflects costly procedures with outcomes poorer than other industrialized nations (Bezruchka, 2012; MacDorman \& Matthews, 2009; Stones \& Arulkumaran, 2014). Caesarean section rates in the US and UK are 32.8 (Martin et al., 2013) and 
24.8 (NHS, 2011), respectively. As having a section appears to be more normative in the US, this may impact birth satisfaction rates. Further, generally and culturally in the UK women expect, and the majority wish, to labor naturally; the majority of maternity units in the UK have birthing units run just by midwives. These differences across the US and UK may address the differences in satisfaction rates based on delivery type across our findings and the Hollins Martin and Martin (2014) findings in the UK. Additional research with standardized data collection procedures with similar sample sizes across US and UK participants is needed to further explore differences across the US and UK. A direct comparison of US and UK birth satisfaction rates in combined datasets using structural equation modeling and including key differences in health service provision and delivery choice options would help researchers better understand these cultural differences.

Further psychometric work (e.g., concurrent and discriminant validity) is needed on the BSS-R in other US samples so that researchers can begin to examine correlates and predictors of birth satisfaction in order to better address childbearing women's intrapartum needs. Additionally, as data is collected in the US, UK, and other countries, researchers can begin to compare birth satisfaction rates across countries in order to address areas of strengths and weaknesses in maternal birth care. The BSS can provide maternal health professionals and researchers with a favorable instrument to quantify childbearing women's birthing satisfaction, which in turn can meet the needs of the "Triple Aim" and heightening the overall patient care experience, therefore, improving overall health and reducing costs.

There are several limitations to this study. Participants were recruited from the Internet and therefore we did not sample those that did not have access to the Internet, did not see the advertisement for the study, or chose not to participate in the study. Recruitment methods 
therefore resulted in select sample of first time mothers. Further research should assess birth satisfaction more generally, and it is our hopes that this scale will be used in the hospital setting. Additionally, this sample consisted of primarily Caucasian, educated, married women who reported that this first birth was planned, therefore, we cannot generalize to all US women. While this study offers a crucial starting point for assessment of the measurement properties of the BSS-R, further research is needed to more fully assess birth satisfaction in a more general and representative US sample.

\section{Conclusion}

The aims of this study were to refine the BSS-R to make it culturally relevant for US participants and examine the factor structure of the scale in a sample of US mothers. Minor wording was changed to make it culturally relevant, and the hypothesized 1-factor (Experience of childbearing) higher-order model containing 3 lower-order factors (Stress, Quality of Care, and Women's attributes) was replicated (Hollins Martin \& Martin, 2014). We then described the level of birth satisfaction in a sample of US mothers, noting what appears to be low quality of care sub-scale scores in our US sample. 


\section{References}

Bezruchka, (2012). The hurrider I go the behinder I get: The deteriorating international ranking of the U.S. health status. Annual Review of Public Health. 33:157-173. doi:10.1146/annurev-publhealth-031811-124649

Brown, T.A. (2006). Confirmatory Factor Analysis for Applied Research. New York: Guilford Press.

Childbirth Connections (2014). Transforming maternity care: What's wrong with the status quo? Retrieved from http://transform.childbirthconnection.org/vision/status-quo/

Clark, S.L., Belfort, M.A., Byrum, S.L., Meyers, J.A., \& Perlin, J.B. (2008). Improved outcomes, fewer cesarean deliveries, and reduced litigation: Results of a new paradigm in patient safety. American Journal of Obstetrics \& Gynecology, 199(2)105.e1-105.e7. doi.org/10.1016/2Fj.ajog.2008.02.031

Declercq ER, Sakala C, Corry MP, Applebaum S, Herrlich, A. (2013). Listening to mothers III: Report of the third national U.S. survey of women's childbearing experiences. New York: Childbirth Connection 2013. Available from http://ransform.childbirthconnection.org/reports/listeningtomothers/

Faul, F., Erdfelder, E., Lang, A.-G., \& Buchner, A. (2007). G*Power 3: A flexible statistical power analysis program for the social, behavioral, and biomedical sciences. Behavior Research Methods, 39, 175-191.

Fleming S., \& Vandermause R. (2011). Grand Multiapare’s evolving experiences of birthing and technology in U.S. hospitals. JOGNN: Journal of Obstetric, Gynecologic, and Neonatal Nursing. 40(6), 742-752.

Furr, M.R. \& Bacharach, V.R. (2014). Psychometrics: An Introduction, (2 ${ }^{\text {nd }}$ ed). Sage: Los 
Angeles.

Hollins Martin, C.J., Snowden, A., \& Martin, C.R. (2012). Concurrent analysis: validation of the domains within the Birth Satisfaction Scale. Journal of Reproductive and Infant Psychology, 30, 247-260.

Hollins Martin, C.J. \& Fleming, V. (2011).The birth satisfaction scale. International Journal of Health Care Quality Assurance, 24, 124-135.

Hollins Martin, C.J. \& Martin, C.R. (2014). Development and psychometric properties of the Birth Satisfaction Scale-Revised (BSS-R). Midwifery, 30, 610-619.

Institute for Healthcare Improvement (2014). The IHI Triple AIM. Retrieved July 1, 2014, from http://www.ihi.org/offerings/Initiatives/TripleAim/Pages/default.aspx

Mahon, P.Y. (1996). An analysis of the concept 'patient satisfaction' as it relates to contemporary nursing care. Journal of Advanced Nursing, 24, 1241-1248.

MacDorman, M. \& Mathews T. (2009). Behind international rankings of infant mortality: How the United States compares with Europe. Hyattsville, MD: National Center Health Statistics.

Martin, J. A., Hamilton, B. E., Osterman, M.J. K., Curtin, S. C., Mathews, T.J. (2013). Births: Final data for 2012. National Vital Statistics Report, 62, 1-102. Hyattsville, MD: National Center for Health Statistics.

Muthén, L. K., \& Muthén, B. O. (1998-2013). Mplus user’s guide (6th ed.). Los Angeles, CA: Muthén \& Muthén.

NHS (2011). Hospital Episode Statistics: NHS Maternity Statistics, 2010-2011. Retrieved from http://www.hscic.gov.uk/catalogue/PUB03071/nhs-mate-eng-2010-2011-rep.pdf.

Renfrew, M., Homer, C., Downe, S., McFadden, A., Muir, N., Prentice, T., et al. (2014). 
Midwifery: An executive summary for The Lancet's series. The Lancet. Published online at http://download.thelancet.com/flatcontentassets/series/midwifery/midwifery_exec_summ.pdf. Sakala, C. (2010). U.S. health care reform legislation offers major new gains to childbearing women and newborns. Birth, 37, 337-340.

Simkin, P. (1991) Just another day in a woman’s life? Women’s long-term perceptions of their first birth experience, Part I, Birth, 18, 4, 203-10.

Stones, W. \& Arulkumaran, A. (2014). Health-care professionals in midwifery care. The Lancet, 384, 1169-1170.

Weir L. \& Andrews, R. (2011, March). The national hospital bill: The most expensive conditions by payer, 2008. HCUP Statistical Brief, 107. Retrieved from http://www.hcup-us.ahrq.gov/reports/statbriefs/sb107.jsp 
Table 1. Standardized factor loadings for the Birth Satisfaction Scale-Revised (BSS-R) 1-factor higher-order model containing 3 lower-order factors $(\mathrm{N}=181)$.

Lower-order factors

Stress Quality Women's

of care attributes

The delivery room staff encouraged me to make decisions

about how I wanted my birth to progress

I found giving birth a distressing experience $(\mathrm{R})$

I came through childbirth virtually unharmed

.64

I felt very anxious during my labour/labor and birth (R)

I felt out of control during my birth experience (R)

I was not distressed at all during labour/labor

.75

I thought my labour/labor was excessively long (R)

.53

I felt well supported by staff during my labour/labor and

birth

The staff communicated well with me during labour/labor

The delivery room was clean and hygienic

.46

Stress

\section{Higher-order factor loadings}

Experience of

childbearing

Quality of care

.87

Women's attributes

.64

Note. $(\mathrm{R})$ = reverse-coded items 